\title{
A NEW INTRAVASCULAR COMPARTMENT: ENDOTHELIAL GLYCOCALIX. ARE THERE CONSEQUENCES FOR THE CLINICAL PRACTICE?
}

\author{
Ioana Grigoraş \\ University of Medicine and Pharmacy “Gr.T. Popa” Iaşi, Romania \\ Anesthesia and Intensive Care Department \\ Regional Institute of Oncology, Iaş i, Romania
}

\begin{abstract}
SHORT TITLE: Endothelial glycocalix in clinical practice
HOW TO CITE: Grigoraş I. A new intravascular compartment: endothelial glycocalix. Are there consequences for the clinical practice? Jurnalul de chirurgie (Iaşi). 2013; 9(4): 311-313. DOI: 10.7438/1584-9341-9-4-1.
\end{abstract}

The glycocalix was first described in 1963 by Bennett as a polysaccharidic layer, which covers the endothelial cells [1]. Long time it was hard to evidence this anatomical structure and it was mainly supposed by its functional consequences. These difficulties are related to the fragile nature of this layer being rapidly destroyed by tissue preparation and to the flow dependency, cultured endothelial cells displaying poor glycocalix. Modern in vitro or in vivo techniques allowed glycocalix visualization and research.

Today glycocalix is considered a bulky, intravascular compartment, which plays an important role in the homeostasis of the vascular wall, blood flow and tissue exchange regulation. It is the first barrier between the intravascular space and cells. Thus, the intravascular space consists of 3 compartments: cellular volume (mainly erythrocytes), plasma volume and the glycocalix volume.

The glycocalix structure is complex. It may be described as a gel with a negative electrical charge that "lubricates" the internal side of vessels. At electron microscopic view it looks like a "brush" with a height of $60-110 \mathrm{~nm}$ lining the vascular endothelium inside [1]. It is composed of an array of macromolecules anchored to the endothelial cells: glycoproteins, proteoglycans and glycosaminoglycans, but also contains plasma proteins (albumin, fibrinogen), enzymes (e.g., lipoprotein lipase, superoxide dismutase) and growth factors (e.g. vascular endothelial growth factor). This entire structure is soaked with water. Glycocalix and endothelial cells form together a morpho-functional structure that is called "endothelial glycocalix layer" [1].

The glycocalix physiology was extensively studied in last decades and new and complex functions are recently described. The main functions described so far are regulation of vascular permeability, mediation of shear stress and prevention of leukocytes and platelets adhesion [1].

The glycocalix pathology is uncovered parallel to its physiology. Several injury factors result in anatomical and/or functional impaiment. Partial alteration (compaction, fragmentation and shedding) consists of selective enzymatic cleavage by heparinase or hyaluronidase. This phenomenon is

Received date: 21.08 .2013

Accepted date: 31.08 .2013

Correspondence to: Ioana Grigoraş, MD, $\mathrm{PhD}$

Head of Anesthesia and Intensive Care Department

Regional Institute of Oncology, Iaşi, Romania

General Henry Mathias Berthlot Street, No. 2-4, 700483, Iaşi, Romania

Phone: 0040 (0) 3742788 10; 0040 (0) 3742788 11;

Fax: 0040 (0) 374278802

E-mail: grigorasioana@yahoo.com 
accompanied by increased plasma level of heparan, condroitin and other glycocalix constituents [2,3]. Injury factors are ischemia / reperfusion, hypoxia / reoxygenation, inflammatory cytokines and proteases and atrial natriuretic peptide [4]. Consequences of glycocalix injury are increased capillary permeability (capillary leak syndrome), tissue edema, enhanced inflammation, hypercoagulability, loss of vascular tone regulation [1].

\section{The revised Starling theory}

According to the "classical" Starling theory the capillary water exchange is governed by the difference between hydrostatic and colloid-osmotic pressure of the intravascular and the interstitial space. Water shifts out at the arteriolar and in at the venular end of capillaries. A small part of the interstitial fluid is drained to the lymphatic circulation.

Anatomical and functional glycocalix description resulted in the review of the Starling theory [5]. The barrier to the interstitial space is represented not only by the endothelial cells, but by the morphofunctional complex called "endothelial glycocalix layer". According to the revised theory water exchange is governed by the difference between hydrostatic and colloidosmotic pressure between the vascular lumen and subglycocalix space devoided of protein $[1,5,6]$. Colloid-osmotic pressure of the interstitial space does not play a role in the water movements. Thus, the rate of water filtration at capillary level is lower than predicted by the classical Starling theory and the major route for water return into intravascular space is lymph circulation [5].

\section{Perioperative volume therapy and glycocalix theory}

The intraoperative permissive administration of volume solutions is based on the concept of "third space" formation by water shift from the intravascular to interstitial space. But modern research showed that "third space" does not exist. Then the interstitial edema is the cause or the consequence of "vigorous2 volume administration?

According to glycocalix theory, there are two types of water movements during and after major surgery [6]. Type I, always present, is physiological water and electrolyte shift devoided of proteins from the intravascular to the interstitial space by an intact vascular barrier. Excessive intraoperative administration of cristalloid solutions will increase the volume of water passing into interstitium. Type II, inconstantly present, is pathological water, electrolytes and macromolecules (proteins or colloids) shift through an altered vascular barrier. It depends on the location, extent and duration of surgery. It is a result of glycocalix degradation with two major iatrogenic causes. On one hand, surgery induces the glycocalix injury by mechanical stress, endotoxin exposure, ischemia / reperfusion. On the other hand, anesthesia is commonly associated with excessive volume administration. Hypervolemia stimulates the secretion of atrial natriuretic peptide, which causes glycocalix degradation with several clinically significant consequences: increased vascular permeability, tissue edema, increased platelet aggregation [6].

This new theory of perioperative water movements provides a logical basis for volume therapy. Thus, minimizing type I water shifts may be accomplished by intraoperative cristalloid administration to replace urinary and insensible losses and colloid administration to replace blood loss [6].

Minimizing type II shifts may result from prevention of glycocalix degradation. To accomplish these goals surgery should be minimally invasive, with gentle visceral manipulation, mechanical sutures, atraumatic dissection and anesthesia may include a neuraxial block and avoid hypervolemia [6].

In conclusion, perioperative volume therapy should not be governed by the cristalloid / colloid or restrictive / liberal controversy, but by the cristalloids and 
colloids administration in optimal amounts with optimal timing to minimize side effects and to preserve the integrity of the glycocalix.

\section{CONFLICT OF INTERESTS}

None to declare.

\section{REFERENCES}

1. Becker BF, Chappell D, Bruegger D, Annecke $\mathrm{T}$, Jacob M. Therapeutic strategies targeting the endothelial glycocalyx: acute deficits, but great potential. Cardiovasc Res. 2010; 87(2): 300-310.

2. Rehm M, Bruegger D, Christ $F$, et al. Shedding of the endothelial glycocalyx in patients undergoing major vascular surgery with global and regional ischemia. Circulation. 2007; 116(17): 1896-1906.

3. Bruegger $\mathrm{D}$, Rehm $\mathrm{M}$, Abicht $\mathrm{J}$, et al. Shedding of the endothelial glycocalyx during cardiac surgery: on-pump versus off-pump coronary artery bypass graft surgery. $J$ Thorac Cardiovasc Surg. 2009; 138(6): 1445-1447.

4. Doherty M, Buggy DJ. Intraoperative fluids: how much is too much? Br J Anaesth. 2012; 109(1): 69-79.

5. Woodcock TE, Woodcock TM. Revised Starling equation and the glycocalyx model of transvascular fluid exchange: an improved paradigm for prescribing intravenous fluid therapy. Br J Anaesth. 2012; 108(3): 384-394.

6. Chappell D, Jacob M, Hofmann-Kiefer K, Conzen P, Rehm M. A rational approach to perioperative fluid management. Anesthesiology. 2008; 109(4): 723-740. 
